\title{
ORDINAL SUM-SETS
}

\author{
MARTIN M. ZUCKERMAN ${ }^{1}$
}

Abstract. A finite set, $B$, of ordinals will be called a sum-set if there are nonzero ordinals $\alpha_{1}, \alpha_{2}, \cdots, \alpha_{n}$ such that the set of sums of $\alpha_{1}, \alpha_{2}, \cdots, \alpha_{n}$, in all $n$ ! permutations of the summands, is $B$. Let $B_{k}$ denote an arbitrary $k$-element sum-set; we consider various matters related to the set of numbers $n$ for which there are $n$ summands for $B_{k}$.

1. In general, addition of ordinals depends on the order of the summands. Various studies, [1], [3], [4], [5], [6], [7], and [8], have been concerned with determining information about the sets $E_{n}$ of natural numbers $k$ for which there exist $n$ (not necessarily distinct) ordinals that in all possible orderings yield $k$ distinct sums. We now investigate the following related problem: We say that a set of $k$ distinct ordinals $\left\{\beta_{1}, \beta_{2}, \cdots, \beta_{k}\right\}$ is a sum-set provided that there are a finite number of (not necessarily distinct) nonzero ordinals $\alpha_{1}, \alpha_{2}, \cdots, \alpha_{n}$ such that the set of sums of $\alpha_{1}, \alpha_{2}, \cdots, \alpha_{n}$ (in all $n$ ! arrangements) is $\left\{\beta_{1}, \beta_{2}, \cdots, \beta_{k}\right\}$. In this case we say that $\alpha_{1}, \alpha_{2}, \cdots, \alpha_{n}$ are summands for $\left\{\beta_{1}, \beta_{2}, \cdots, \beta_{k}\right\}$. Thus, here we are concerned with which finite sets of ordinals are sets of sums of a finite number of ordinals.

2. Every nonzero ordinal $\alpha$ has a unique Cantor normal form:

$$
x=\omega^{\alpha_{1}} M_{1}+\omega^{\alpha_{2}} M_{2}+\cdots+\omega^{\alpha_{r}} M_{r},
$$

where $r, M_{1}, M_{2}, \cdots, M_{r}$ are nonzero natural numbers and where $\alpha_{1}>\alpha_{2}>\cdots>\alpha_{r} \geqq 0 . \alpha_{1}$ is called the degree of $\alpha$, written " $\operatorname{deg}(\alpha)$ ", and $M_{1}$ is called the leading coefficient of $\alpha$.

Every nonzero ordinal $\alpha$ can be uniquely represented in the form

$$
\chi=\omega^{\alpha_{1}} M_{1}+\rho,
$$

Received by the editors September 13, 1971 and, in revised form, January 3, 1972. AMS 1970 subject classifications. Primary 04A10; Secondary 04A20.

Key words and phrases. Ordinal addition, Cantor normal form, permutations of summands.

${ }^{1}$ This research was supported by a City University of New York Faculty Research Award, 1970-1972.

(c) American Mathematical Society 1972 
in which, with reference to $r, M_{1}, M_{2}, \cdots, M_{r}, \alpha_{1}, \alpha_{2}, \cdots, \alpha_{r}$ in (1),

$$
\begin{aligned}
\rho & =0 & & \text { if } r=1 ; \\
& =\omega^{\alpha_{2}} M_{2}+\cdots+\omega^{\alpha_{r}} M_{r} & & \text { otherwise. }
\end{aligned}
$$

We shall refer to (2) as the remainder form representation of $\alpha ; \rho$ will be called the remainder of $\alpha$, written "rem( $(\alpha)$ ". Clearly, if $\rho \neq 0$, then $\operatorname{deg}(\rho)<$ $\alpha_{1}$. For any set $A$ of ordinals, let $R_{A}=\{\operatorname{rem}(\alpha): \alpha \in A\}$.

A set $A$ is said to be compatible if $A$ is a set $\left\{\alpha_{1}, \alpha_{2}, \cdots, \alpha_{k}\right\}$ of nonzero ordinals and if each $\alpha_{i}, 1 \leqq i \leqq k$, has the same degree and leading coefficient. If $A\left(=\left\{\alpha_{1}, \alpha_{2}, \cdots, \alpha_{k}\right\}\right)$ is compatible and if the leading coefficient of each $\alpha_{i}$ is $M$, we say that $A$ is $M$-compatible and that $\operatorname{deg}\left(\alpha_{1}\right)$ is the degree of $A$, written " $\operatorname{deg}(A)$ ".

Throughout this section $B$ will always denote a $k$-element set of ordinals $\left\{\beta_{1}, \beta_{2}, \cdots, \beta_{k}\right\}, k=1,2, \cdots$; moreover, $\beta_{i}=\omega^{\beta_{i, 1}} M_{i}+\rho_{i}$ will be the remainder form representation of $\beta_{i}, i=1,2, \cdots, k$.

Clearly, every unit set $B$ is a sum-set. If $B=\left\{\beta_{1}, \beta_{2}\right\}$ is compatible, we can suppose $\beta_{1}<\beta_{2}$. Then $\beta_{1}$ and $\rho_{2}-\rho_{1}$ are summands for $B$.

For any set $A$, let $\mathscr{P} *(A)$ be the set of nonempty subsets of $A$. If $J \in$ $\mathscr{P} *(\{1,2, \cdots, n\})$, let $\Sigma_{J}$ be the group of permutations of $J$. In particular, if $J=\{1,2, \cdots, n\}$, we write " $\Sigma_{n}$ " instead of " $\Sigma_{J}$ ".

Let $R$ be a set of ordinals. We shall say that $C$ is an additive derivative of $R$ if there exist ordinals $\gamma_{1}, \gamma_{2}, \cdots, \gamma_{m}$ for which

$$
C=\left\{\rho+\sum_{j \in J} \gamma_{\phi(j)}: \rho \in R, J \in \mathscr{P}^{*}(\{1,2, \cdots, m\}), \text { and } \phi \in \Sigma_{j}\right\} .
$$

THEOREM 1. $B$ is a sum-set if and only if for some $l, M$ satisfying $1 \leqq l \leqq$ $M<\infty$,

(i) $B$ is $M$-compatible, and

(ii) $R_{B}$ is an additive derivative of $R_{B_{0}}$ for some l-element subset $B_{0}$ of $B$.

Proof. Suppose $\alpha_{1}, \alpha_{2}, \cdots, \alpha_{n}$ are summands for $B$. Let the remainder form representation of $\alpha_{i}$ be $\alpha_{i}=(1)^{\operatorname{deg}\left(\alpha_{i}\right)} M_{i}+r_{i}$ for $i=1,2, \cdots, n$. Let $\alpha^{*}=\max \left\{\operatorname{deg}\left(\alpha_{i}\right): 1 \leqq i \leqq n\right\}$, let $A=\left\{i: 1 \leqq i \leqq n\right.$ and $\left.\operatorname{deg}\left(\alpha_{i}\right)=\alpha^{*}\right\}$, and let $M=\sum_{i \in A} M_{i}$. Clearly, $A$ has $M$ or fewer elements. For each $\phi \in \Sigma_{n}$, let $j_{\phi}$ be the largest number $i$ for which $\phi(i) \in A$. Then

$$
\sum_{i=1}^{n} \alpha_{\phi(i)}=\omega^{\alpha^{*}} M+r_{j_{\phi}}+\alpha^{\prime},
$$

where

$$
\begin{aligned}
\alpha^{\prime} & =0 & & \text { if } j_{\phi}=n, \\
& =\sum_{i=j \phi^{+1}}^{n} \alpha_{\phi(i)} & & \text { if } j_{\phi}<n .
\end{aligned}
$$


In the latter case, $\operatorname{deg}\left(\alpha^{\prime}\right)<\alpha^{*}$. Therefore, $B=\left\{\sum_{i=1}^{n} \alpha_{\phi(i)}: \phi \in \Sigma_{n}\right\}$ is $M$ compatible.

Let $B_{0}=\left((1)^{\alpha^{*}} M+r_{i} . i \in A\right\}$; clearly, $B_{0}$ is an l-element subset of $B$ for $1 \leqq l \leqq M$. Let $\gamma_{1}, \gamma_{2}, \cdots, \gamma_{m-1}$ be those ordinals among $\alpha_{1}, \alpha_{2}, \cdots, \alpha_{n}$ for which $i \notin A$ (if any such exist); let $\gamma_{m}=0$. According to (3) and (4), $R_{B}$ is an additive derivative of $R_{B_{0}}$.

Now suppose that for $1 \leqq l \leqq M<\infty$, conditions (i) and (ii) hold; we can further suppose that $B_{0}=\left\{\beta_{u_{1}}, \beta_{u_{2}}, \cdots, \beta_{u_{l}}\right\}$ and that $\gamma_{1} \leqq \gamma_{2} \leqq \cdots \leqq$ $\gamma_{m}$ are ordinals for which

$$
R_{B}=\left\{\rho_{u_{i}}+\sum_{j \in J} \gamma_{\phi(j)}: 1 \leqq i \leqq l, J \in \mathscr{P} *(\{1,2, \cdots, m\}) \text { and } \phi \in \Sigma_{J}\right\} .
$$

Surely, $\gamma_{1}=0$. Moreover, for each $J \in \mathscr{P} *(\{1,2, \cdots, m\}) \sim\{1\}$ and for each $\phi \in \Sigma_{J}, \operatorname{deg}\left(\sum_{j \in J} \gamma_{\phi(j)}\right)<\operatorname{deg}(B)$. For $i=1,2, \cdots, l+m-1$, let

$$
\begin{aligned}
\alpha_{i} & =(1)^{\operatorname{deg}(13)}+\rho_{\iota_{i}} & & \text { if } 1 \leqq i<l ; \\
& =(1)^{\operatorname{deg}(l 3)}(M-l+1)+\rho_{\iota_{l}} & & \text { if } i=l ; \\
& =\gamma_{i-l+l} & & \text { if } l<i<l+m .
\end{aligned}
$$

Then $\alpha_{1}, \alpha_{2}, \cdots, \alpha_{l, \ldots 1}$ are summands for $B$.

THEOREM 2. Every compatible set B can be extended to a sum-set.

Proof. Let $B$ be $M$-compatible. Consider the set $B^{*}$ for which $\omega^{\mathrm{deg}(B)} M, \rho_{1}, \rho_{2}, \cdots: \rho_{k}$ are the summands. Surely, $B \subseteq B^{*}$.

3. For each nonzero natural number $k$, let $B_{k}$ denote an arbitrary $k$ element sum-set, and let $N\left(B_{k}\right)$ be the set of natural numbers $n$ for which there are $n$ (not necessarily distinct) summands for $B_{k}$. For positive natural numbers $k$ and $M$, let $N_{\text {int }}(k, M)=\bigcap\left\{N\left(B_{k}\right)\right.$ :all $M$-compatible $\left.B_{k}\right\}$; let $N_{\max }(k, M)=\max \left\{N\left(B_{k}\right)\right.$ :all $M$-compatible $\left.B_{k}\right\}$ (if this maximum exists); let $N_{\min }(k, M)=\min \left\{N\left(B_{k}\right)\right.$ :all $M$-compatible $\left.B_{k}\right\}$.

THEOREM 3. For all nonzero natural numbers $k$ and $M, N_{\max }(k, M)$ exists and

$$
N_{\text {max }}(k, M)=k+M-1 .
$$

Proof. For any $k, M$, consider $\beta_{i}=(1) M+(i-1), \quad l=1,2, \cdots, k$. Let $\alpha_{i}=(1)$ for $1 \leqq i \leqq M$ and, if $k>1$, let $\alpha_{i}=1$ for $M+1 \leqq i \leqq M+k-1$. Then $\alpha_{1}, \alpha_{2}, \cdots, \psi_{1 /, 1}$ are summands for $\left\{\beta_{1}, \beta_{2}, \cdots, \dot{\beta}_{k}\right\}$. Thus $N_{\text {max }}(k, M) \geqq k+M-1$.

Let $\left\{\gamma_{1}, \gamma_{2}^{\prime}, \cdots, \gamma_{k} ;\right.$ be an arbitrary $k$-element $M$-compatible set. Let the remainder form representation of $\gamma_{i}$ be $\gamma_{i}=(1)^{\gamma} M+\rho_{i}, i=1,2$, $\cdots, k$, where $\rho_{1}<\rho_{2}<\cdots<\rho_{k}$. Suppose $\delta_{1}, \delta_{2}, \cdots, \delta_{L}$ are summands 
for $\left\{\gamma_{1}, \gamma_{2}, \cdots, \gamma_{k}\right\}$. At least one and at most $M$ of these $\delta_{i}$ are of degree $\gamma$. For some $j, 1 \leqq j \leqq L$, suppose $\operatorname{deg}\left(\delta_{j}\right)=\gamma$; let $\rho_{j}=\operatorname{rem}\left(\delta_{j}\right)$. For any nonzero natural number $r$, let $0<\sigma_{1}<\sigma_{2}<\cdots<\sigma_{r}<\omega^{\gamma}$. Then the ordinals $\omega^{\gamma} M+\rho_{j}, \omega^{\gamma} M+\rho_{j}+\sigma_{1}, \omega^{\gamma} M+\rho_{j}+\sigma_{1}+\sigma_{2}, \cdots,(1)^{\gamma} M+\rho_{j}+\sigma_{1}+\sigma_{2}+\cdots$ $+\sigma_{r}$ are all distinct. Thus there are at most $k-1$ summands for $\left\{\gamma_{1}, \gamma_{2}, \cdots\right.$, $\gamma_{k}$ \} of degree less than $\gamma$. Consequently, $L \leqq M+k-1$, and $N_{\max }(k, M)=$ $k+M-1$.

THEOREM 4 . Let $M \geqq 1$.

(a) For all $B_{1}, N\left(B_{1}\right)=\{1,2, \cdots, M\}$;

(b) for all $B_{2}, N\left(B_{2}\right)=\{2,3, \cdots, M+1\}$;

(c) for $3 \leqq k \leqq M, N_{\text {int }}(k, M)=N\left(\left\{(1) M+2^{i-1}: i=1,2, \cdots, k\right\}\right)=$ $\{k, k+1, \cdots, M\}$.

Proof. For $k=1,2, \cdots$, assume $M \geqq k$; let $B_{k}=\left\{\beta_{1}, \beta_{2}, \cdots, \beta_{k}\right\}$, where $\beta_{i}={ }_{(1)}^{\beta} M+\rho_{i}$ is the remainder form representation of $\beta_{i}, i=1,2$, $\cdots, k$. For $k \leqq j \leqq M$, let $\alpha_{i}=(1)^{\beta}+\rho_{i}$ for $1 \leqq i<j$ and let $\alpha_{j}=(1)^{\beta}(M-j+1)+$ $\rho_{j}$. Clearly, for each such $j, \alpha_{1}, \alpha_{2}, \cdots, \alpha_{j}$ are summands for $B_{k}$; hence $\{k, k+1, \cdots, M\} \subseteq N\left(B_{k}\right)$ for each $B_{k}$.

(a) and (b). For $k=2$, assume $\beta_{1}<\beta_{2}$. For $M=1,2, \cdots$, let $\gamma_{1}=$ $\gamma_{2}=\cdots=\gamma_{1 I}=(1)^{\beta}+\rho_{1}$ and let $\gamma_{M I+1}=\rho_{2}-\rho_{1}$; then $\gamma_{1}, \gamma_{2}, \cdots, \gamma_{. I+1}$ are summands for $B_{2}$. Theorem 3 now guarantees that $N\left(B_{1}\right)=\{1,2, \cdots, M\}$ and $N\left(B_{2}\right)=\{2,3, \cdots, M+1\}$.

(c). In order to show that for $3 \leqq k \leqq M, N_{\text {int }}(k, M)=\{k, k+1, \cdots, M\}$, it suffices to show that $N\left(\left\{(1) M+2^{i-1}: i=1,2, \cdots, k\right\} \subseteq\{k, k+1, \cdots, M\}-\right.$ hence $=\{k, k+1, \cdots, M\}$, by the above. Let $\gamma_{1}, \alpha_{2}, \cdots, \alpha_{n}$ be summands for $\left\{a, M+2^{i-1}: i=1,2, \cdots, k\right\}$.

Suppose that at least one of the $\alpha_{i}-$ say $\alpha_{n_{1}}$ is finite. Clearly, at least one of the $\alpha_{i}$ must be infinite.

Case 1. There are at least two distinct remainders among the infinite $\alpha_{i}$. Then one of these must be 1 ; another is of the form $2^{l}$ for $l \geqq 1$. Then $1+\alpha_{n}=2^{\prime \prime \prime}$ for $m \geqq 1$; therefore $\alpha_{n}$ is odd. Also, $2^{l}+\alpha_{n}=2^{p}$ for some $p \geqq 1$; therefore $\kappa_{\text {" }}$ is even. Contradiction!

Case 2. All of the infinite $\alpha_{i}$ have the same remainder, $\rho$. Then $\rho$ must be 1 . Let $j$ be the number of infinite $x_{i}$; we can suppose $\alpha_{j: 1} \leqq \alpha_{j+2} \leqq$ $\cdots \leqq x_{11}<(1)$. We must have $\alpha_{j+1}=1$ in order to yield the sum (1) $M+2$. Similarly, we must have $j+2 \leqq n$ and $x_{j .2}$ equal to 2 or 3 --in order to yield the sum (1) $M+4$. But either of these choices for $x$, : would yield a sum-(1) $M+3$ or (1) $M+5-$ that is not in $\left\{(1) M+2^{i}: i=1,2, \cdots, k\right\}$.

Thus all of the $\alpha_{i}$ must be infinite. If $n>M$, then since each $\alpha_{i}$ is of degree 1 , the leading coefficient of any sum is at least $n$-hence is bigger than $M$. If $n<k$, then there are fewer than $k$ remainders obtained among the $n$ ! permutations. 
Corollary. If $\gamma_{i}=(1) M+2^{i-1}, i=1,2, \cdots, k$, and $k \geqq \max (3, M+1)$, then $\left\{\gamma_{1}, \gamma_{2}, \cdots, \gamma_{k}\right\}$ is not a sum-set.

4. The determination of $N_{\min }(k, M)$ is considerably more difficult! It is closely related to problems encountered in [7].

THEOREM 5. Let $k, M$, and $m$ be arbitrary nonzero natural numbers. Then $N_{\min }(k, M+m) \leqq N_{\min }(k, M)$.

Proof. Let $\beta_{i}=\omega^{\beta} M+\rho_{i}$ be the remainder form representation of $\beta_{i}, i=1,2, \cdots, k$. Suppose that $\alpha_{1}, \alpha_{2}, \cdots, \alpha_{n}$ are summands for the $k$-element set $\left\{\beta_{1}, \beta_{2}, \cdots, \beta_{k}\right\}$. At least one of the ordinals $\alpha_{i}, 1 \leqq i \leqq n$, is of degree $\beta$; let $\alpha_{i_{0}}$ be any such ordinal and let the remainder form representation of $\alpha_{i_{0}}$ be $\alpha_{i_{0}}=\omega^{\beta} M_{i_{0}}+r_{i_{0}}$. Let $\alpha_{i_{0}}^{*}=\omega^{\beta}\left(M_{i_{0}}+m\right)+r_{i_{0}}$, and for $i \neq i_{0}$ and $1 \leqq i \leqq n$, let $\alpha_{i}^{*}=\alpha_{i}$. Let $\beta_{i}^{*}=\omega^{\beta}(M+m)+\rho_{i}, i=1,2, \cdots, k$. Then $\alpha_{1}^{*}, \alpha_{2}^{*}, \cdots, \alpha_{n}^{*}$ are summands for the $k$-element set $\left\{\beta_{1}^{*}, \beta_{2}^{*}, \cdots, \beta_{k}^{*}\right\}$.

For each nonzero natural number $k$ we let

$$
N_{\text {min }}(k)=\min \left\{N_{\min }(k, M): M=1,2, \cdots\right\} .
$$

We note that $N_{\text {min }}(k)=N_{\text {min }}(k, 1)$ for $1 \leqq k \leqq 449$. (See [3], [4], [5, pp. 265-266], and [7, proofs of Theorems 2, 3, and 4].) Theorems 3, 5, and 6 together imply that for all natural numbers $k$ and $M$, except for $k=1$, $M=1$ and for $k=2, M=1$,

$$
N_{\min }(k, M)<N_{\max }(k, M) .
$$

THEOREM 6. Let $k$ and $n$ be nonzero natural numbers. Let

$$
\begin{array}{r}
L(n)=\max \left\{l:(l+2)^{2} 2^{l}-4(l+2) \leqq(l+1) 2^{n-l}\right\} . \\
\text { If } k \leqq(L(n)+2) 2^{n \cdot 2}-2^{L(n)}+1, \text { then } N_{\min }(k) \leqq N_{\min }(k, 1) \leqq n .
\end{array}
$$

Proof. This follows from [7, Theorem 2].

For example, if $n=6$, then $L(n)=2$; Theorem 6 indicates that for $k \leqq$ $61, N_{\min }(k) \leqq 6$.

For each nonzero natural number $n$, the maximum number $m_{n}$ of distinct values that can be assumed by a sum of $n$ nonzero ordinals in all $n$ ! permutations of the summands has been calculated in [1] and [5]. The numbers $m_{n}$ increase with $n$; for $n \geqq 3, m_{n}<n$ !. It is easily seen from the formulas given by Erdös and Wakulicz that for $n \geqq 10, n \neq 14$,

$$
m_{r^{\prime}}=3^{4(k-(l-1))-3(1-l)} 11^{1-l} 193^{l-1},
$$

where $n=5 k+l$ for $k, /$ nonnegative integers with $l \leqq 4$, and where for nonnegative integers $r$ and $s$,

$$
\begin{aligned}
r \dot{-} & =r-s, & & r \geqq s, \\
& =0, & & r<s .
\end{aligned}
$$


It immediately follows that if $k>m_{n}$, then $N_{\min }(k)>n$. Moreover, [3], [4], [5], [6], and [7, Theorem 4] yield that for $1 \leqq k \leqq 29$ and for $31 \leqq k \leqq$ 449 , if $n$ is such that $m_{n-1}<k \leqq m_{n}$, then $N_{\min }(k)=n$.

We note that $N_{\min }(29)=N_{\min }(31)=5$, whereas $N_{\min }(30)=6$; thus $N_{\min }$ is not monotonic. Furthermore, [8, Theorem 1] indicates that there are infinitely many $k$ for which there are $j$ and $l$ satisfying $j<k<l$ and $N_{\min }(j)=N_{\min }(l)<N_{\min }(k)$.

Let $n \geqq 1$. Let

$$
\begin{aligned}
& \mathscr{B}_{n}=\{\left\{\left\langle l_{1}, r_{1}, 1\right\rangle,\left\langle l_{2}, r_{2}, 2\right\rangle, \cdots,\left\langle l_{n}, r_{n}, n\right\rangle\right\}: \\
& r_{1}=0 \text { and for } i=1,2, \cdots, n-1, l_{i} \geqq 1, \text { and } r_{i+1}=r_{i} \text { or } \\
&\left.r_{i+1}=r_{i}+1\right\} .
\end{aligned}
$$

For each $B \in \mathscr{B}_{n}$ and for each $j=0,1, \cdots, n-1$, let $B_{j}=\left\{\left\langle l_{i}, i\right\rangle:\left\langle l_{i}, j, i\right\rangle \in\right.$ $B\}$ and let $B_{j}^{\#}$ be the number of distinct sums $\sum l_{i}$ such that $\left\langle l_{i}, i\right\rangle \in B_{j}^{\prime} \cup$ $B_{j}^{\prime \prime}$, where $B_{j}^{\prime}$ ranges over nonempty subsets of $B_{j}$ and $B_{j}^{\prime \prime}$ ranges over subsets (possibly empty) of $\bigcup\left\{B_{u}: u \neq j\right.$ and $\left.0 \leqq u \leqq n-1\right\}$. Let $B^{\#}=\sum_{j=0}^{n-1} B_{j}^{\#}$, let $\mathscr{C}_{n}=\bigcup\left\{B^{\#}: B \in \mathscr{B}_{n}\right\}$, and let $\mathscr{D}_{n}=\left\{1+\bar{x}: x \in \mathscr{C}_{n}\right\}$.

THEOREM 7. Let $k$ and $n$ be nonzero natural numbers. If there exist nonzero natural numbers $n_{1}, n_{2}, \cdots, n_{s} ; k_{1}, k_{2}, \cdots, k_{s}(s \geqq 1)$ such that $n=$ $\sum_{i=1}^{s} n_{i}$ and $k=\prod_{i=1}^{s} k_{i}$, where $k_{1} \leqq n_{1}$ and for $i=2,3, \cdots, s, k_{i} \in \mathscr{D}_{n_{i}}$, then $N_{\min }\left(k, n_{1}\right) \leqq n$.

Proof. See the proof of $[7$, Theorem 1].

THEOREM 8. Let $k, m$, and $M$ be nonzero natural numbers.

(a) Let $s \in \mathscr{D}_{m}$. Then

In particular,

$$
N_{\min }(s k, M) \leqq m+N_{\min }(k, M)
$$

$$
N_{\min }(2 k, M) \leqq 1+N_{\min }(k, M)
$$

(b) Let $1 \leqq s \leqq m$. Then for some $M^{\prime}, M<M^{\prime} \leqq 2 M$,

$$
N_{\min }\left(s k, M^{\prime}\right) \leqq m+N_{\min }(k, M) .
$$

Proof. This follows from [7, Theorem 3].

THEOREM 9. Let $k$ and $M$ be nonzero natural numbers. Then for some $M^{\prime}, M<M^{\prime} \leqq 2 M$,

$$
N_{\min }\left(k+1, M^{\prime}\right) \leqq 1+N_{\min }(k, M)
$$

Proof. This follows from [7, Theorem 4]. 


\section{REFERENCES}

1. P. Erdös, Some remarks on set theory, Proc. Amer. Math. Soc. 1 (1950), 127-141. MR 12, 14.

2. W. Sierpiński, Cardinal and ordinal numbers, 1st ed., Monografie Mat., Tom 34, PWN, Warsaw, 1958. MR 20 \#2288.

3. - Sur les séries infinies de nombres ordinaux, Fund. Math. 36 (1949), 248-253. MR 12, 14.

4. A. Wakulicz, Sur les sommes de quatre nombres ordinaux, Soc. Sic. Lett. Varsovie. C. R. Cl. III. Sci. Math. Phys. 42 (1949), 23-28. MR 13, 923.

5. - Sur la somme d'un nombre fini de nombres ordinaux, Fund. Math. 36 (1949), 254-266. MR 12, 14; 13, 1138.

6. - Correction au travail "Sur les sommes d'un nombre fini de nombres ordinaux", Fund. Math. 38 (1951), 239.

7. M. M. Zuckerman, Sums of at most 8 ordinals, Z. Math. Logikgrundlagen Math. (to appear).

8. _- Sums of at least 9 ordinals, Notre Dame J. Formal Logic (to appear).

Department of Mathematics, City College of the City University of New YORK, NEW YORK, NEW YORK 10031 